\title{
Design of Portable Integrated Suitcase Weighing Scale Displaying Weight on Smart Phone via Bluetooth Interface
}

\author{
Ujwala N. Petigara \\ PG Student, Embedded System \\ G.H. Patel College of Engineering \\ Vallabh Vidyanagar, India
}

\author{
Bhaskar Thakker, Ph.D \\ H.O.D. of EC Department \\ G.H. Patel College of Engineering \\ Vallabh Vidyanagar, India
}

\begin{abstract}
Design \& implementation of a Traveler's Bag having built in weighing scale for the identification of weight of the bag with wireless communication capability is presented here. The bag encompass a bottom support member for holding the system and a pair of wheels secured to the bottom support member. The bag is equipped with load cell mounted on the bottom part over the wheels. The LCD display displaying weight is mounted near the handle of bag for easy reading of weight. The whole electronic system is installed such that the normal usage of a traveler for the bag is not suffered. Built-in scale is also designed to equip with Bluetooth Communication Interface which can transmit the weight information to the cell phone using Bluetooth Interface without intervention of user for loading the bag on the conveyor belt at Airport. The Android Application developed for a cell phone provide, smart communication between Bag and System. Bluetooth Communication Interface to transmit the weight to Android Application which is saved over Internet Storage makes this application really useful for the future check -in- process at airports. Ultra-low power series MSP430 Microcontroller is considered as a energy efficient and cost effective solution in this system.
\end{abstract}

\section{General Terms}

Portable weighing scale, Android Application

\section{Keywords}

Load Cell, Sigma Delta ,MSP430, Bluetooth Interfacing.

\section{INTRODUCTION}

Now a day's travellers are become more concerned about their Baggage weight, specially who travels by air. Due to many reasons airlines put the restriction on carrying no. of bags and the baggage weight by passenger. All airlines have implemented checked luggage policies that restrict the weight luggage containers to a certain maximum weight per bag. Different airlines offer their own weight limit, maximum weight limit allowed by airlines is around $30 \mathrm{kgs}$ per bag. And there are usually stiff monetary penalties imposed on travellers whose luggage exceeds these limitations. Typically, luggage weight is determined at the ticket counter when passengers and their baggage are checked in for travel .If a piece of luggage exceeds the weight limit the airlines will allow travelers to redistribute the contents from suitcases that are over the predetermined weight to ones that are under that weight or must leave various articles to reduce the weight commonly by simply discarding items or by placing items in their personal vehicle or a locker to be retrieved on their return. This is extraordinarily inconvenient and potentially costly as well as slows down the check-in procedure for all the other individuals behind the person whose luggage is too heavy. It would be advantageous for a traveller to know the weight of their luggage prior to arriving at their point of departure. This can be accomplished by placing the packed suitcases on a conventional scale of the digital type with digital displays showing numbers to a traveller. This procedure would be time consuming however, as the individual performing the packing would constantly have to weigh and re-weigh the suitcase until a desired weight is achieved. It would be much more advantageous, and convenient, to know the weight of the suitcase as it is being filled with luggage.

\section{LITERATURE SURVEY}

Various types of weighing scales exist that allow a user to measure the weight of their luggage. Some require the luggage to be placed on the scale and others require the scale to be affixed to the object. Individuals have continuing concerns about the weight of the luggage they may be carrying, whether due to weight restrictions enforced at an airport for a flight or a person not wanting to overstress carrying too heavy luggage and there needs to be an easy and reliable way to measure the weight of luggage without having to look for a scale or having to find it and then attach it to the object. Some Patents and research paper are available to study this concept, some of them are discussed below.

In David et al [3] presents the luggage bag having built-inscale which slides into and out of a base of bag. Basically there are two load cell are used, one sensor is to weigh the baggage load and another for user weight measurement. There is one slide mechanical switch which allows the base to be in two particular position. Thus in a closed position the scale is disposed within the base, so that the user can measure the weight of the baggage. In a open position the scale is slide out of base ,that is external to the base allowing user to weigh him or herself. Conclusion can be made that this system offers ,a RFID communication interface -which sends the weight of bag to some other RFID reader which is more innovative part presented in this article. This design adding complexities to design and expense due to two ,as there are two different load sensors to operate system in two different working mode . 


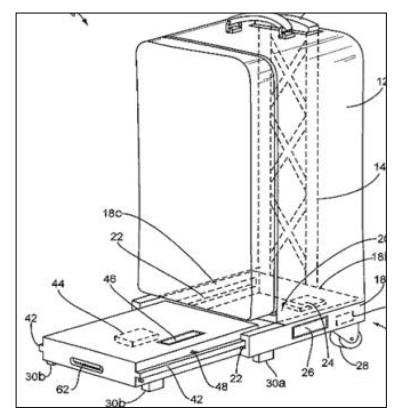

Fig 1: Perspective views of an article of luggage [1]

In Dahikar et al [2] This paper helps to understand that how weighing scale can be design taking care of filtering of noise at ADC input. In particular, the focus is on using digital filtering techniques to remove measurement noises from the extremely low frequency noise of the static weighing system. In this paper it is also discussed that why to use sigma delta ADC with load sensor to achieve noise free conditioning at analog input end which is an very important block of any weighing scale system. In addition it is explained that how an integrated PGA helps to eliminate the need of an external signal conditioning circuitry. In Andreas et al [3] this application note presented the benefits of using MSP430 series microcontroller in the design of portable weighing scale. Also explained how to configure the integrated sigma delta ADC with internal programmable gain amplifier to achieve required resolution of weight measurement.

\section{PROPOSED SYSTEM}

\subsection{System Block Diagram}

Traveler's Bag with Weighing Scale system is designed here with aim to provide accurate weight information to user. Fig. 2 shows various functional blocks involved in this system. It mainly includes a microcontroller, a Load Cell, Low Pass Filter, LCD Display, Bluetooth Module, Battery , and a Switch. Microcontroller is playing a major role in the system and all other peripheral modules are interfaced with it. Load cell is used to sense the weight to be measured which gives electrical signal as output which is then after filtering the noise picked-up from environment is passed to differential inputs of sigma delta ADC of MSP430 microcontroller.

Thus microcontroller work on ADC output to give proper value which can be displayed on Segmented LCD display as a weight of bag. Each functional block of the proposed system can be described briefly as follows

\subsection{System Features}

Operation of system is going to be divided into three parts. Basic features related to the operation of overall system are described as below.

\subsubsection{Tare Mode}

The tare function allows the mass of a substance to be measured separately from the mass of its container. When the TARE button is pressed, the scale measures the mass and records it. This reading is subtracted from each subsequent measurement

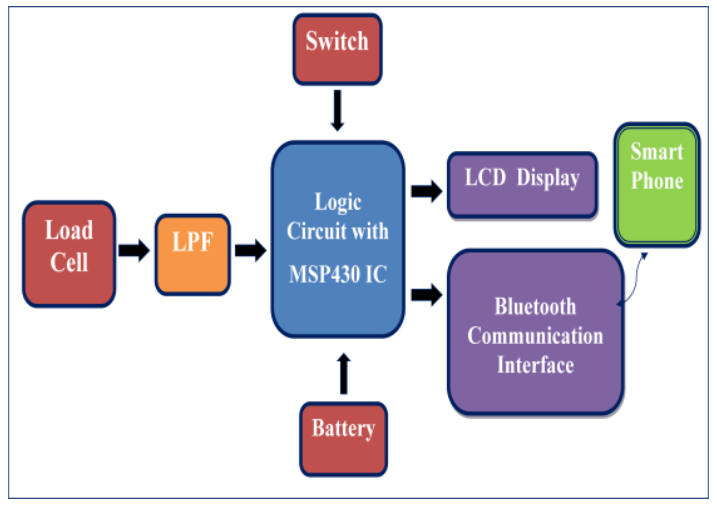

Fig 2: System Block Diagram

\subsubsection{Calibration Mode}

Pressing CAL Push-Button to initiates the two-point calibration sequence. To prevent the calibration data loss on power removal, data is saved on flash memory

\subsubsection{Weight Measurement Mode}

By default the system works in weight measurement mode -as long as the calibration point are correct .If not system enters into calibration mode, where user needs to do the calibration manually by following the above calibration procedure. In this mode system reads the ADC count ,process that count to display it on LCD as a weight of load.

\subsubsection{Bluetooth Interface Mode}

In this mode system receives command from user's cell phone Android application. In response to this command system transmit the weight of Bag serially using USART via Bluetooth Interface available on system using HC-05 bluetooth module

\subsection{Load Cell Selection}

Load cell is a passive, electrical type transducer which converts a force into an electrical signal. Load cell uses different operating principles like load cell based on piezoelectric effect ,fluid pressure, on elasticity, on magnetostriction effect. However, the load cells based on strain gages are most popular and the reason is their characteristics. Load cell of Electric output, Bending-Beam type having Hermetic seal Type is being selected for this work. In market load cells are available with above configuration in various range like $10 \mathrm{~kg}$, $20 \mathrm{~kg}, 40 \mathrm{~kg}$,maximum $2000 \mathrm{~kg}$. Here load cell choice can be made particularly for bags which are supposed to carry maximum weight limit defined by different airlines. Presently the maximum weight limit is $32 \mathrm{~kg}$ defined by airline; thus load cell of $40 \mathrm{~kg}$ is appropriate for this application.

\section{IMPLEMENTATION}

The proposed system has been implemented in IAR Embedded Workbench IDE with C-SPY debugger which supports MSP430 series microcontroller. Load cell having part No.CZL601-40kg and MSP430fg479 IC ,having Sigma Delta ADC along with PGA is used to verify the response of load cell at 3 Volt DC excitation voltage.

Bluetooth Module HC-05, is a serial port ,class -2 Bluetooth module with complete $2.4 \mathrm{GHz}$ radio transceiver and 
baseband. Offer UART interface with programmable baud rate with programmable and $-80 \mathrm{dBm}$ sensitivity .

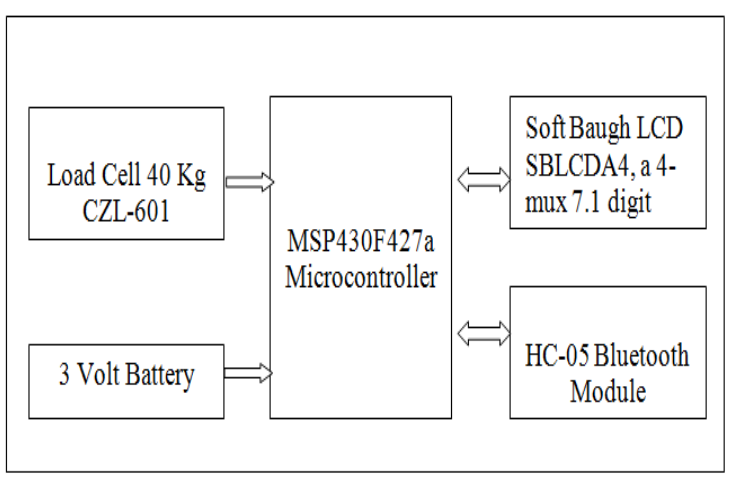

Fig 3 Block Diagram of System Board with Selected components

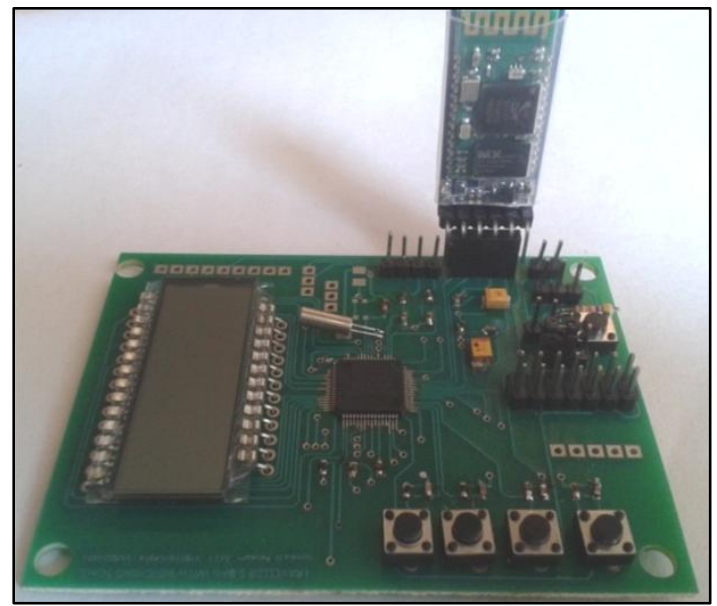

Fig. 4 Image of System Board

The response of Load Cell which is having excitation voltage $3 \mathrm{~V}$ DC and SD16 ADC count is depicted with the help of below graph in Fig. 5 The load cell output voltage characteristic is almost linear for given values of mass in grams .

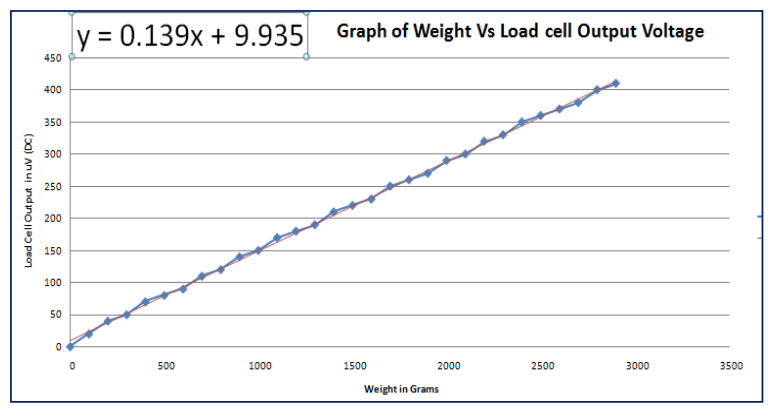

Fig: 5 Graph of Sigma Delta ADC Count Vs. Weight

\subsection{Android Application Development}

Travellers Bag with Weighing Scale is equipped to have Bluetooth Communication using Android app. Thus Android app is an another important feature of this thesis work, where smart phone users can easily use it to access the weight of their Bag .In addition this application, also saves the record of
Weight with Date and Time, which can be accessed by user. One more exclusive feature of this application is that the weight of bag can be stored as a file on Cloud over Internet connection for further access by Airport Check-in Counter process. Thus this application going to rid-off the drawn out in check-in process at Airport for user. Figures below showing the result for this Androi Application.

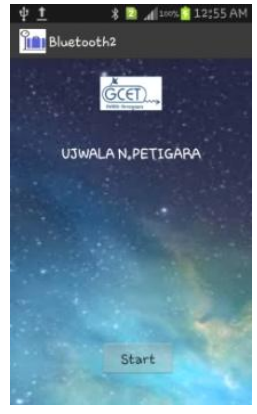

Fig 6 Snapshot of Android Splash Activity

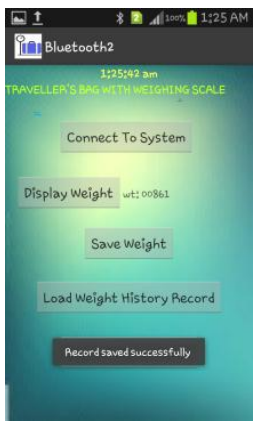

Fig.8 Snapshot of Android Main Activity showing Response against Save Weight Button click event

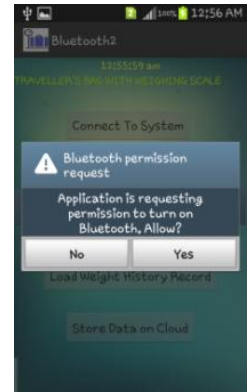

Fig.7 Snapshot of Android Main Activity to connect with Scale System via Bluetooth

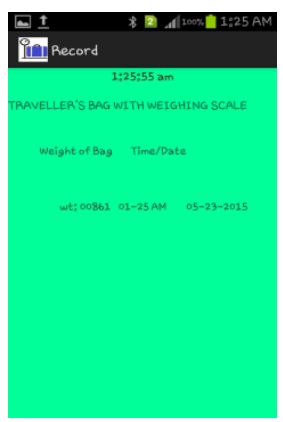

Fig.9 Snapshot of Android Record Response against Load Weight History Record Buttons click event Activity showing

\section{CONCLUSION}

This paper presents the weighing scale to be integrated inside a traveler's bag. Portable weighing scale design is possible using a single chip solution with a MSP430FX4X series ICs by Texas Instruments. The advantage of using MSP430 series Prototype for Travelers Bag with Weighing Scale has been achieved with the help of MSP430f427a microcontroller along with Load Cell ,Segmented LCD Display, Bluetooth Module, and Android Application. This design works on merely 3Volt Battery supply. The prototype hardware design successfully tested for various conditions. Weighing Scale designed here giving resolution of $5 \mathrm{~g}$ weight; which is enough for the present requirement. And scale is designed to weigh the maximum load $35 \mathrm{Kg}$. for user. Cell Phone Android Application has been working successfully for displaying weight as well saving the weight with timestamp record history in SQLite database in Android for further access of user. In addition saving weight of bag as a file over Internet source will be really helpful for future set-up at Airport for accessing weight of passengers bag on-line. 


\section{REFERENCES}

[1] David Falk "Luggage having a built-in scale configured to slide into and out of the luggage base, where the scale can measure weight in either configuration", US 8344271 B1, Jan. 2013.

[2] Dr. Dahikar and Mr. Patle " Design of an Embedded Platform for Digital Weighing System to Enhance Measuring Capabilities" IJIRCCE Paper ISSN(Online): 2320-9801,ISSN (Print): 2320-9798, Oct. 2013 Published by : Publication Date: Apr. 2010

[3] Andreas Dannenberg "MSP430F42x Single Chip Weigh Scale " slaa220 Texas Instrument,Nov.2004
[4] Load Cell Details available at http://www.omega.co.uk/prodinfo/load -cells.html

[5] LCD DISPLAY Specification available at www.softbaugh.com

[6] MSP430 MICROCONTROLLER BASICS by John Davis ,Elsevier Academic Press, : ISBN: 978-0-75068276-3.

[7] MSP430FG47x Mixed Signal Microcontroller Data Sheet (SLAS580D )

[8] MSP430x4xx Family User's Guide (SLAU056L)

[9] MSP-TS430PN80 PCB BOARD USER GUIDE (SLAU278T) 